\title{
Cognitive behavior therapy with youth and health care reform: a congenial union
}

\begin{abstract}
This short opinion paper discusses cognitive behavioral therapy (CBT) with youth in the era of health care reform. The commentary addresses the ways CBT is consistent with health care reform imperatives. Further, CBT's focus on accountability, credentialing, early intervention, and interdisciplinary collaboration is emphasized.
\end{abstract}

CBT with youth; Health care reform
Volume I Issue 2 - 2014

\author{
Robert D Friedberg, Maxwell Berlyant \\ Center for the Study and Treatment of Anxious Youth at Palo \\ Alto University, USA
}

Correspondence: Robert D Friedberg, Center for the Study and Treatment of Anxious Youth, Palo Alto University, |79| Arastrasdero Rd., Palo Alto, CA. 94022, USA, Tel 650-96I7503(17),Email rfriedberg@paloaltou.edu

Received: May 31, 2014 | Published: June 09, 2014

Abbreviations: CBT, cognitive behavioral therapy; ACA, affordability and accountability act; $\mathrm{PHC}$, primary health care; BHC,

\section{Introduction}

Recent health care changes (e.g. Affordability and Accountability Act, ACA) firmly embed behavioral health care into primary health care (PHC) and offer unprecedented parity for behavioral health care (BHC) delivery. ${ }^{1}$ Further, integration with PHC helps ensure BHC's sustainability. With these opportunities come greater responsibilities for accountability, early intervention/prevention, and collaboration with other health care professionals. Fortunately, CBT with children and adolescents represents a good standard for treating depression, anxiety, obsessive-compulsive disorder, anger/aggressive behavior, and PTSD. ${ }^{2}$ This short commentary emphasizes the ways cognitive behaviorally (CBT) oriented clinicians are equipped to meet the emerging trends and demands in their care of young patients.

\section{Accountability}

The ACA clearly places a high value on clinical accountability. The ACA asserts that quality indicators are essential. ${ }^{1,3}$ Indeed, monitoring patient outcomes is key to accountable clinical practice. Obtaining good clinical outcome is essential to patients, payers, and practitioners alike. $^{4}$

Good outcomes begin with competent providers. While there is ample supply of mental health practitioners, there is a serious lack of professionals genuinely trained and competent to deliver empirically based treatment to children. ${ }^{5,6}$ Practitioners should possess knowledge of and clinical skills with evidence based methods. Second, they must receive good supervision from a supervisor well-schooled in these approaches. Third, monitoring the on-going use of these practices and their outcomes is required.

Outcome metrics and quality indicators are long-standing staples in CBT spectrum approaches with youth. There is a seamless integration of assessment and treatment during young patient's course of care. CBT oriented clinicians use these signposts to guide intervention decisions on whether to apply low/high intensity interventions, change treatment plans, lengthen/shorten duration of care, and/or refer to another provider.

\section{Credentialing}

Good training and supervision facilitates transfer from efficacy studies completed in methodologically rigorous lab studies to effectiveness in real world clinical settings. While no absolute consensus for credentialing in CBT exists, guiding standards abide. In order to ensure CBT with youth is provided with treatment integrity, we recommend that trainers, consultants, and supervisors receive specialty training post-doctorally and/or post-licensure at one of the many recognized CBT centers. Additionally, supervisors should possess first-hand knowledge of their trainees 'patients through either live supervision or viewing audio/videotape. Finally, sessions need to be regularly evaluated with a cognitive behavioral rating scale.

Finally, proper training is a way to ensure program developers and evaluators avoid Type III errors. ${ }^{7}$ As readers recall from elementary statistics, Type I error reflects erroneously finding true differences where none exist whereas Type II error is the failure to find true differences where one legitimately occurs. Type III error is failing to correctly execute an intervention as planned and/or with fidelity resulting in no effect of treatment. ${ }^{7}$ Simply, if you compare poorly delivered CBT with another comparison treatment, fail to find a difference, and then conclude these two paradigms are equivalent, you are making a Type III error. The failure for CBT to outperform the other treatments is due to improper implementation. To use a pharmacological analogy, the proper dose of CBT was not received. Effective training and supervision produce competent clinicians who provide impactful CBT.

\section{Early intervention and prevention}

Early intervention (EI), prevention, and other innovative delivery systems are highly valued by the ACA. At risk children's psychopathological trajectories are derailed and existing vulnerabilities are quieted by EI. Many EI programs are rooted in cognitive behavioral procedures. ${ }^{8}$ Internet, computer, and smart phone applications are also developing. ${ }^{9}$ Finally, non-traditional formats such as camps for young people where procedures are implemented intensely over a week-long periods are emerging. ${ }^{10}$

\section{Coordination with primary care: less lone rangers, more justice league members}

Coordination with other health care professionals is another ACA imperative. Communication with others requires abandoning silo mentalities and embracing a team approach. True collaboration does not just happen; it takes work. Behavioral health care providers must increase their fluency with medical terminology, recognition of 
contextual issues related to primary care service delivery and become more comfortable delivering interventions in shorter sessions with young patients (e.g. 20-30 minutes). ${ }^{11}$

CBT approaches are well-suited to all these demands. Numerous articles describe efficient and efficacious protocols for clinical problems encountered in pediatric practice. ${ }^{12}$ Indeed, there is growing evidence that supports co-locating empirically based services directly in pediatric offices. ${ }^{13}$ As CBT clinicians we must continue to develop efficient methods suitable for treating pediatric problems.

A brief metaphor illustrates the central points. The Lone Ranger is a legendary fictional masked crime fighter in American west who battles evil singly or with his one colleague, Tonto. More recent heroes such as Superman, Batman, Wonder Woman, and Flash unite in the Justice League to combat their common foes. In order to remain ready, willing, and able to join forces in health reform, mental health professionals must shed the often held identities as Lone Rangers solely capable of caring for young people with behavioral health problems. Rather, we should seek increased community with other health care professionals and act more like Justice League members who conjointly battle common pediatric behavioral health problems.

\section{Conclusion}

Behavioral health care is poised at the precipice during the current earth-shattering health care changes in the United States. Modular CBT is well-equipped to deliver the kind of accountable care demanded by the ACA. In order to meet the clinical challenges and make the most of emerging opportunities, clinicians are well-advised to obtain training from supervisors genuinely credentialed in CBT and work assiduously to deliver an effective dose of accountable care to young patients.

The new health care era is not without risks and challenges. We will need to diligently advocate for access and coverage for our most vulnerable populations. Efforts will need to be directed at improving patients' mental health literacy. Additionally, we must make sure that CBT with youth is genuinely delivered by specialists who are competently trained in the approach. Finally, continued outcome research and model development is needed to create innovative procedures so non-responders are effectively treated.

\section{Acknowledgments}

None.

\section{Conflicts of interest}

Author declares there are no conflicts of interest.

\section{Funding}

None.

\section{References}

1. Emanuel EJ. Reinventing American Health Care. Perseus, New York, USA. 2014

2. March JS. The future of psychotherapy for mentally ill children and adolescents. J Child Psychol Psychiatry. 2009;50(1-2):170-179.

3. Hoagwood KE, Olin SS, Horwitz S, et al. Scaling up evidence-based practices for children and families in New York State: Toward Evidencebased policies on implementation for State Mental Health Systems. J Clin Child Adolesc Psychol. 2014;43(2):145-157.

4. Nordahl KC. Healthcare reform: Implications for independent practice. Professional Psychology: Research and Practice. 2012;43(6):535-544.

5. Beidas RS, Koerner K, Weingardt KR, et al. Training research: Practical recommendations for maximum impact. Adm Policy Ment Health. 2011;38(4):223-237.

6. Shafran R, Clark DM, Fairburn CG, et al. Mind the gap: Improving the dissemination of CBT. Behav Res Ther. 2009;47(11):902-909.

7. Hasson H. Systematic evaluation of implementation fidelity of complex intervention in health and social care. Implement Sci. 2010;5:67.

8. Friedberg RD, Brelsford GM, Pearl A, et al. Preventing anxiety and depression in children. In: A Columbus (Ed.), Community and environmental psychology. Nova Press, New York, USA. 2011. p. 179-190.

9. Berry RR, Lai B. The emerging role of technology in cognitivebehavioral therapy for anxious youth. Journal of Rational-Emotive \& Cognitive Behavior Therapy. 2014;32(1):57-66.

10. Santucci LC, Ehrenreich-May J. A randomized controlled trial of the child anxiety multi-day program (CAMP) for separation anxiety disorder. Child Psychiatry Hum Dev. 2013;44(3):439-451.

11. Kelly JF, Coons HL. Integrated healthcare and professional psychology: is the setting right for you? Professional Psychology: Research and Practice. 2012;43(6):586-595.

12. Weersing VR, Rozenman MS, Maher-Bridge M, et al. Anxiety, depression, and somatic distress: Developing a transdiagnostic toolbox for pediatric practice. Cogn Behav Pract. 2012;19(1):68-82.

13. Kolko DJ, Perrin E. The integration of behavioral health interventions in children's health care: services, science, and suggestions. J Clin Child Adolesc Psychol. 2014;43(2):216-228. 